\title{
Terapêuticas utilizadas em recém-nascidos com malformações congênitas internados em unidade neonatal
}

\author{
Therapies for neonates with congenital malformations admitted to a neonatal unit
}

Terapéuticas utilizadas en recién nacidos con malformaciones congénitas internados en unidad neonatal

Maria Vera Lúcia Moreira Leitão Cardoso ${ }^{1}$, Valéria Rocha Mendes Lima ${ }^{2}$, Fabíola Chaves Fontoura ${ }^{3}$, Sofia Esmeraldo Rodrigues ${ }^{4}$, Isadora Andrade Saraiva ${ }^{5}$, Fernanda Cavalcante Fontenele ${ }^{6}$

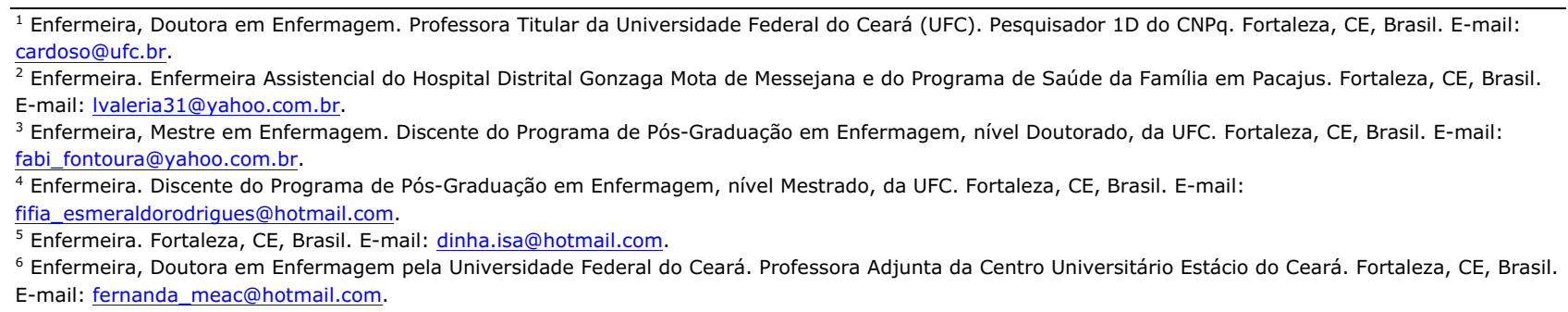

\section{RESUMO}

Objetivou-se caracterizar as terapêuticas aplicadas aos recém-nascidos com malformações congênitas internados em Unidade Neonatal e identificar se existe associação entre as terapêuticas utilizadas e o tipo de malformação. Estudo descritivo, prospectivo, quantitativo realizado em instituição pública de Fortaleza - CE, Brasil. A coleta de dados foi realizada por meio dos registros dos prontuários de 30 neonatos com malformações congênitas. Verificou-se que a ocorrência de malformações foi maior no sexo feminino, independente da idade materna, idade gestacional ou peso ao nascer; prevaleceram as malformações do sistema nervoso central e osteomuscular. As terapêuticas utilizadas variaram conforme a evolução clínica do neonato. Os dados não demonstraram significância estatística quando associada à variável malformação congênita e às terapêuticas utilizadas $(p>0,05)$. As terapêuticas não estão diretamente relacionadas ao tipo de malformação, mas ao quadro clínico do neonato.

Descritores: Recém-nascido; Anormalidades Congênitas; Enfermagem Neonatal; Terapêutica.

\section{ABSTRACT}

The aim of this study was to characterize the treatments applied to newborns with congenital malformation hospitalized in a neonatal unit and to identify whether there is an association among the treatments used and the type of malformation. A descriptive, prospective and quantitative study was developed in a public institution in Fortaleza, Ceará, Brazil. Data were collected using the medical records of 30 neonates with congenital malformations. The incidence of malformations was higher among females, regardless of the mother's age, gestational age or weight at birth; malformations of the central nervous and musculoskeletal systems prevailed. The treatments used varied according to the clinical evolution of the neonate. The data collected did not present statistical significance when associated with the variable of congenital malformation and the treatments used $(p>0.05)$. The treatments are not directly related to the type of malformation, but to the clinical condition of the neonate. Descriptors: Infant, Newborn; Congenital Abnormalities; Neonatal Nursing; Therapeutics.

\section{RESUMEN}

Se objetivó caracterizar las terapéuticas aplicadas a recién nacidos con malformaciones congénitas internados en Unidad Neonatal e identificar existencia de asociación entre las terapéuticas utilizadas y el tipo de malformación. Estudio descriptivo, prospectivo, cuantitativo, realizado en institución pública de Fortaleza-CE-Brasil. Datos recolectados mediante registro de historias clínicas de 30 neonatos con malformaciones congénitas. Se verificó que las malformaciones fueron prevalentes en el sexo femenino, independientemente de la edad materna, edad gestacional o peso al nacer; prevalecieron las malformaciones del sistema nervioso central y osteomuscular. Las terapéuticas utilizadas variaron conforme la evolución clínica del neonato. Los datos no expresaron significatividad estadística al asociárselos con la variable malformación congénita y las terapéuticas utilizadas $(p>0,05)$. Las terapéuticas no están relacionadas directamente al tipo de malformación, sino al cuadro clínico del neonato.

Descriptores: Recién Nacido; Congenital Abnormalities; Enfermería Neonatal; Terapéutica. 


\section{INTRODUÇÃO}

Malformação Congênita (MC) pode ser definida como toda anomalia funcional ou estrutural do desenvolvimento do feto, decorrente de fator originado antes do nascimento, seja genético, ambiental ou desconhecido, mesmo quando o defeito não é aparente no RecémNascido (RN) e somente manifestar-se tardiamente ${ }^{(1)}$.

Diversos fatores, internos ou externos, podem contribuir para o aparecimento de um defeito genético, no entanto, a causa é desconhecida na maioria dos casos. Ressalta-se que além da mortalidade, as malformações são responsáveis, também, pelo alto índice de morbidade, risco para desenvolvimento de complicações clínicas, com números de internamentos e gravidade das intercorrências ${ }^{(2)}$. Todos os anos, em $6 \%$ dos nascimentos em todo o mundo, cerca de 7,9 milhões de crianças nascem com defeitos congênitos graves. Destes, quase 3,2 milhões ficam deficientes para toda vida, o que irá necessitar de acompanhamento clínico ${ }^{(3)}$. As populações estão expostas aos riscos de desenvolverem malformações congênitas, porém a frequência e o tipo variam de acordo com a raça, a etnia e as condições socioeconômicas ${ }^{(4)}$.

Para melhoria organizacional da categorização das malformações congênitas existentes, ocorreu a décima revisão da Classificação Internacional de Doenças pela Organização Mundial da Saúde (OMS), resultando nas seguintes categorias: malformações do olho, do ouvido, da face e do pescoço; do aparelho circulatório; do aparelho respiratório; fenda labial e/ou fenda palatina; outras malformações congênitas do aparelho digestivo; malformações congênitas dos órgãos genitais; do aparelho urinário; do aparelho osteomuscular; outras malformações congênitas; e anomalias cromossômicas ${ }^{(5)}$.

O nascimento de um RN pré-termo (RNPT) ou com alguma MC necessita de internamento em local com recursos tecnológicos, humanos e terapêuticos especializados, a fim de proporcionar cuidados mais complexos. Para tanto, esses suportes são encontrados em Unidades de Terapias Intensivas Neonatais (UTIN), cuja assistência ocorre continuamente, e cujo surgimento tem proporcionado aumento na sobrevida orgânica dos prematuros, principalmente os que apresentam extremo baixo peso(6).

As UTIN são unidades formalmente instituídas, organizadas e voltadas conscientemente para um saber profissional que requer especificidade, habilitação e princípios voltados ao objeto do cuidado, ou seja, o RNPT ou doente, estando os malformados inseridos nesse contexto $^{(7)}$. O aumento da sobrevida dos recém-nascidos com malformações melhorou consideravelmente nos últimos anos e coincidiu com o avanço de técnicas cirúrgicas e novos recursos terapêuticos, o que têm contribuído para correção de anomalias operáveis (ou cirurgicamente corrigíveis) ${ }^{(8)}$.

A hospitalização e, consequentemente, o cuidado intensivo implementado aos recém-nascidos em unidades neonatais estão associados à submissão destes a um número excessivo de procedimentos, como as punções venosas, as sondagens orogástricas e vesicais, as glicemias capilares, a realização de curativos, a aspiração de vias aéreas, a entubação endotraqueal, retiradas de drenos, dentre outros, o que pode gerar desconforto, estresse e dor ${ }^{(6,9)}$.

Em face ao exposto, o estudo mostra-se relevante, por contribuir para prática da assistência de enfermagem neonatal individualizada, acerca de informações pertinentes à terapêutica utilizada durante o internamento de recém-nascidos com malformação congênita, de forma mais detalhada; e oportunizar o conhecimento de profissionais da saúde sobre essa clientela tão específica, o que irá subsidiar estudos posteriores, para que o cuidado de Enfermagem ao RN com malformação seja mais amplo.

Sabendo-se que os recém-nascidos malformados, uma vez internados nessas unidades, necessitarão de terapêuticas que visam manter a integridade da saúde, questionou-se: quais terapêuticas são implementadas na Unidade Neonatal junto aos neonatos que nascem com malformação congênita? Existe associação entre as terapêuticas implementadas ao RN com o tipo de malformação apresentada, conforme a classificação do CID-10?

Assim, objetivou-se caracterizar as terapêuticas aplicadas aos recém-nascidos com malformações congênitas internados em Unidade Neonatal e identificar se existe associação entre as terapêuticas utilizadas e o tipo de malformação.

\section{MÉTODOS}

Estudo exploratório, descritivo, prospectivo e quantitativo. Ressalta-se que este estudo é recorte de uma pesquisa maior intitulada "Recém-nascidos internados na unidade neonatal: ênfase na avaliação da dor e na prevalência da malformação congênita", financiada pelo Conselho Nacional de Desenvolvimento Científico e Tecnológico (CNPq), conforme processo 483352/2011-0. Foi realizado em Unidades de Internações Neonatais 1 e 2, em instituição pública do município de Fortaleza/CE, Brasil, referência no Estado. 
Os setores dispunham de 54 leitos, 21 de alto risco e o restante para unidade de médio risco.

A população constou de todos os recém-nascidos que apresentaram algum tipo de malformação congênita visível ou não ao nascimento, durante o período da coleta de dados, realizada de novembro de 2011 a janeiro de 2012, os quais foram encaminhados à Unidade Neonatal, de alto ou médio risco, resultando em uma amostra de 30 RN.

Foram incluídos os recém-nascidos com qualquer anomalia congênita, internados na Unidade Neonatal que nasceram na instituição pesquisada ou transferidos de outras instituições durante o período de coleta de dados, com peso maior que 500 gramas, independente da idade gestacional, sexo, estatura, número da gestação, tipo de parto, apgar, cor, diagnóstico, modalidade de oxigenoterapia, terapia medicamentosa e forma de nutrição.

Os critérios de exclusão foram os recém-nascidos malformados, com óbito constatado imediatamente à avaliação do neonatologista na sala de parto, não sendo necessária sua transferência para a Unidade Neonatal.

A coleta de dados foi realizada a partir dos registros dos prontuários de neonatos com malformações congênitas, por meio de formulário, contendo variáveis como: idade materna, idade gestacional, sexo, peso, tipo de malformação congênita. Em acréscimo apresentava outras variáveis que caracterizavam as seguintes terapêuticas: modalidade ventilatória; forma de nutrição; terapia medicamentosa; curativo; acesso venoso; intervalos de tempo de manuseio para realização do balanço hídrico; realização de cirurgia.

Os dados foram organizados, ordenados e avaliados conforme tipo de variável no Programa Excel e analisados consoante o Statistical Pacckage for the Social Sciences $\left(\right.$ SPSS $^{\circledR}$ ) versão 20. Utilizada análise bivariada para se avaliar a associação entre a categoria de malformação com as variáveis relacionadas à oxigenoterapia, à nutrição, ao balanço hídrico, ao curativo (local e tipo de cobertura), à cirurgia, ao acesso venoso e à terapia medicamentosa.

A estatística descritiva com frequência simples e absoluta, medidas de dispersão, como média e desvio padrão foi aplicada quando conveniente. Na estatística inferencial, utilizou o teste Qui-quadrado $\left(x^{2}\right)$ para verificar associação das variáveis categóricas e o teste de correlação de Spearman'srho para as variáveis quantitativas, em que para ambos se considerou nível de significância de $5 \%(p<0,05)$.
Para classificação das malformações congênitas, foi utilizada a Classificação Estatística Internacional de Doenças e Problemas Relacionados à Saúde, décima revisão (CID-10), que fornece não somente códigos relativos a doenças, como também variedade de sinais e sintomas causados pelas mesmas.

O projeto foi aprovado pelo Comitê de Ética da instituição, conforme parecer número 546/2011, sendo respeitados os aspectos éticos que envolvem pesquisas com seres humanos, de acordo com o que preceitua a Resolução 196/96, do Conselho Nacional de Saúde. Os pais ou responsáveis pelos recém-nascidos malformados assinaram o Termo de Consentimento Livre e Esclarecido, autorizando a participação dos mesmos no estudo.

\section{RESULTADOS}

No intuito de melhor descrever os resultados alcançados, foram elaboradas tabelas, em que estão discriminadas as variáveis relevantes para o estudo e seus respectivos valores, em conformidade aos testes executados de acordo com a amostra estudada. Foram analisados 30 recém-nascidos na instituição pesquisada, com predominância do sexo feminino, 19 (63,3\%), com idade gestacional variando de 31 a 42 semanas, sendo a média de $36,7 \pm 2,7$ semanas. O peso médio ao nascer foi de $2,726 \mathrm{~g}$.

Dentre as variáveis maternas, verificou-se que a idade variou de 19 a 40 anos, sendo que a maioria foi de 20-30 anos, 18 (60\%), com média de 24,6 anos. O parto abdominal foi presente em 18 RN (60\%) da amostra.

Em conformidade à classificação elaborada pelo CID10, foram evidenciadas 48 malformações congênitas em 29 recém-nascidos, podendo apresentar-se isoladas ou associadas em cada RN. Em um recém-nascido identificou-se a micrognatia, que apesar de ter sido registrada como malformação no prontuário, não está classificada no capítulo XVII do CID -10, por isso não está na Tabela 2. Como foi um único caso, optou-se em não excluí-la do estudo, o que resultou em uma amostra de 30 recém-nascidos. Entretanto, constataram-se com maior frequência: 18 casos $(37,5 \%)$ do total das malformações de anomalias do sistema osteomuscular, predominando pé torto congênito $(33,3 \%)$ e outras malformações $(22,2 \%)$, incluindo o nanismo tanatotrófico, ausência de fíbula, crânio assimétrico; $11(22,9 \%)$ malformações do sistema nervoso central (SNC), lideradas por mielomeningocele $(45,4 \%)$, seguida pela hidrocefalia $(36,4 \%)$; e fenda labial e/ou palatina, com cinco casos. As malformações menos acometidas foram as referentes ao aparelho respiratório e às 
anomalias cromossômicas, ambas com $4,1 \%$, dentre o total diagnosticado.

Quanto à terapêutica implementada aos recémnascidos na Unidade Neonatal, as Tabelas 3 e 4 especificam a diversidade e frequência desta, consoante à amostra do estudo.

Tabela 1: Perfil dos recém-nascidos acometidos por malformações congênitas. Fortaleza, CE, Brasil, 2012.

\begin{tabular}{|c|c|c|c|c|}
\hline Variáveis & $\mathbf{N}$ & $\%$ & Média & DP \\
\hline \multicolumn{5}{|l|}{ Sexo } \\
\hline Feminino & 19,0 & 63,3 & & \\
\hline Masculino & 11,0 & 36,7 & & \\
\hline \multicolumn{5}{|l|}{ Peso ao nascer (gramas) } \\
\hline$<999$ & 2,0 & 6,6 & 2726 & 985,4 \\
\hline $1000-1499$ & 1,0 & 3,4 & & \\
\hline $1500-2499$ & 11,0 & 36,7 & & \\
\hline $2500-3999$ & 13,0 & 43,3 & & \\
\hline$>4000$ & 3,0 & 10,0 & & \\
\hline \multicolumn{5}{|l|}{ Idade Gestacional (semanas) } \\
\hline$<30$ & 1,0 & 3,4 & 36,7 & 2,7 \\
\hline $30-34$ & 7,0 & 23,3 & & \\
\hline $35-36,9$ & 7,0 & 23,3 & & \\
\hline $37-42$ & 15,0 & 50,0 & & \\
\hline \multicolumn{5}{|l|}{ Tipo de Parto } \\
\hline Abdominal & 18,0 & 60,0 & & \\
\hline Vaginal & 12,0 & 40,0 & & \\
\hline \multicolumn{5}{|l|}{ Idade Materna (anos) } \\
\hline$\leq 19$ & 6,0 & 20,0 & 24,6 & \\
\hline $20-30$ & 18,0 & 60,0 & & \\
\hline $31-40$ & 6,0 & 20,0 & & \\
\hline
\end{tabular}

Tabela 2: Categoria de malformações congênitas apresentadas por recém-nascidos internados na Unidade Neonatal. Fortaleza, CE, Brasil, 2012.

\begin{tabular}{|c|c|c|}
\hline Categorias das malformações congênitas & $\mathbf{N}$ & $\%$ \\
\hline \multicolumn{3}{|l|}{ Sistema osteomuscular $(n=18)$} \\
\hline Pé torto congênito & 5,0 & 27,8 \\
\hline Outros & 4,0 & 22,2 \\
\hline Gastrosquise & 3,0 & 16,7 \\
\hline Onfalocele & 2,0 & 11,1 \\
\hline Hérnia diafragmática & 2,0 & 11,1 \\
\hline Macrocefalia & 2,0 & 11,1 \\
\hline \multicolumn{3}{|l|}{ Sistema nervoso central $(n=11)$} \\
\hline Mielomeningocele & 5,0 & 45,4 \\
\hline Hidrocefalia & 4,0 & 36,4 \\
\hline Espinha bífida c/ hidrocefalia & 1,0 & 9,1 \\
\hline Encefalocele & 1,0 & 9,1 \\
\hline Fenda labial e/ou palatina $(n=5)$ & 5,0 & 100,0 \\
\hline \multicolumn{3}{|l|}{ Aparelho circulatório $(n=4)$} \\
\hline Malformação na válvula tricúspide & 1,0 & 25,0 \\
\hline Ventrículo único & 1,0 & 25,0 \\
\hline Ventrículomegalia & 1,0 & 25,0 \\
\hline Cardiopatia & 1,0 & 25,0 \\
\hline \multicolumn{3}{|l|}{ Olho, ouvido, face e pescoço $(n=5)$} \\
\hline Orelhas displásicas & 2,0 & 40,0 \\
\hline Deformidades nos dedos das mãos & 1,0 & 20,0 \\
\hline Malformação no pavilhão auricular & 1,0 & 20,0 \\
\hline Macroglossia & 1,0 & 20,0 \\
\hline \multicolumn{3}{|l|}{ Outras malformações do aparelho digestivo $(n=3)$} \\
\hline Atresia de esôfago & 2,0 & 66,7 \\
\hline Atresia duodenal & 1,0 & 33,3 \\
\hline \multicolumn{3}{|l|}{ Anomalias cromossômicas $(n=2)$} \\
\hline Síndrome de Down & 2,0 & 100,0 \\
\hline
\end{tabular}


Tabela 3: Terapêuticas aplicadas em recém-nascidos portadores de malformações congênitas. Fortaleza, CE, Brasil, 2012.

\begin{tabular}{|c|c|c|}
\hline Variáveis $(n=30)$ & $\mathbf{N}$ & $\%$ \\
\hline \multicolumn{3}{|l|}{ Oxigenoterapia } \\
\hline Ar ambiente & 14,0 & 46,7 \\
\hline Ventilação Mecânica & 6,0 & 20,0 \\
\hline Oxi-hood & 6,0 & 20,0 \\
\hline CPAP* & 4,0 & 13,3 \\
\hline \multicolumn{3}{|l|}{ Terapia medicamentosa } \\
\hline Antibióticos & 15,0 & 50,0 \\
\hline Sem medicação & 7,0 & 23,3 \\
\hline Antibióticos + Hidratação venosa & 3,0 & 10,0 \\
\hline Hidratação venosa & 2,0 & 6,7 \\
\hline Opióides & 1,0 & 3,3 \\
\hline Hidratação venosa + medicação oral & 1,0 & 3,3 \\
\hline Antibióticos + opióides + Hidratação venosa & 1,0 & 3,3 \\
\hline \multicolumn{3}{|l|}{ Nutrição } \\
\hline Dieta por Sonda orogástrica & 11,0 & 36,7 \\
\hline Nutrição parenteral & 8,0 & 26,7 \\
\hline Dieta via oral & 7,0 & 23,3 \\
\hline Dieta zero & 4,0 & 13,3 \\
\hline \multicolumn{3}{|l|}{ Cirurgia } \\
\hline Não & 19,0 & 63,3 \\
\hline Aguarda & 7,0 & 23,3 \\
\hline Sim & 4,0 & 13,3 \\
\hline \multicolumn{3}{|l|}{ Intervalo de balanço Hídrico } \\
\hline $3 / 3 h$ & 23,0 & 76,7 \\
\hline $2 / 2 h$ & 7,0 & 23,3 \\
\hline
\end{tabular}

* Continuous Positive Airway Pressure

Tabela 4: Terapêuticas referentes aos curativos aplicados em recém-nascidos portadores de malformações congênitas. Fortaleza, CE, Brasil, 2012.

\begin{tabular}{|c|c|c|}
\hline Variáveis $(n=30)$ & $\mathbf{N}$ & $\%$ \\
\hline \multicolumn{3}{|l|}{ Curativo } \\
\hline Não & 19,0 & 63,3 \\
\hline Sim & 11,0 & 36,7 \\
\hline \multicolumn{3}{|l|}{ Local do Curativo $(n=11)$} \\
\hline Região Sacral & 6,0 & 54,5 \\
\hline Cicatriz Umbilical & 3,0 & 27,3 \\
\hline Região Abdominal & 2,0 & 18,2 \\
\hline \multicolumn{3}{|l|}{ Cobertura $(n=11)$} \\
\hline Gaze + Colagenase+ SF 0,9\% & 3,0 & 27,3 \\
\hline Cobertura Estéril & 2,0 & 18,2 \\
\hline Sem Especificação & 2,0 & 18,2 \\
\hline Gaze + Colagenase & 1,0 & 9,1 \\
\hline Sulf. Prata $1 \%$ & 1,0 & 9,1 \\
\hline Gaze+ Sulf. Prata 1\% + Álcool 70\% & 1,0 & 9,1 \\
\hline Gaze & 1,0 & 9,1 \\
\hline
\end{tabular}

A Tabela 3 apresenta a distribuição das terapêuticas utilizadas nos recém-nascidos malformados, segundo as variáveis investigadas.

Observou-se que $53,3 \%$ dos neonatos foram submetidos à oxigenioterapia. Quanto à terapêutica medicamentosa aplicada aos recém-nascidos, percebeuse a predominância do uso somente de antibióticos (ATB) $50,0 \%$ (15); 10,0\% (3) receberam ATB associada à hidratação venosa (HV); 3,3\% (1) em ATB com hidratação venosa e opióides; e 23,3\% (7) que não receberam nenhum tipo de medicamento. Os demais utilizaram terapia com opióides, medicação oral ou HV isoladamente.

Quanto à terapia nutricional, a dieta administrada por gavagem por meio da sonda orogástrica (SOG) sobressaiu, com 36,7\% (11) dentre as formas de nutrição dos recém-nascidos internados na unidade neonatal, seguida da nutrição parenteral, 26,7\% (8).

Do total da amostra pesquisada, $63,3 \%$ (19) não realizaram e/ou não necessitavam de cirurgia, entretanto $13,3 \%$ (4) foram submetidos a algum procedimento cirúrgico relacionado à malformação apresentada ao nascimento, durante o período da coleta dos dados.

Quanto à frequência de realização do balanço hídrico, $76,7 \%$ (23) dos recém-nascidos eram manuseados com intervalos de $3 / 3$ horas, de forma a manter assistência rigorosa, visando manutenção do equilíbrio hemodinâmico do RN.

Em relação à apresentação de curativos associados a alguma malformação, 11 recém-nascidos foram sinalizados, perfazendo total de $36,7 \%$ da amostra 
investigada. Os demais não apresentaram curativos específicos em conformidade ao diagnóstico, porém utilizaram dispositivos, como cateteres venosos centrais, com película protetora aderida à pele, não sendo contabilizados e registrados para o estudo.

Dentre os 11 curativos registrados, a maioria era localizada na região sacral, com 54,5\% (6) dos casos, seguido da cicatriz umbilical, com $27,3 \%$ (3). Como cobertura mais utilizada sobressaiu a gaze associada à Colagenase e Solução Fisiológica a 0,9\%, com 27,3\% (3) dos curativos.

Ao investigar possíveis associações entre a variável malformação com as variáveis oxigenoterapia, forma de nutrição, terapia medicamentosa, tipo de acesso venoso, intervalo na realização do balanço hídrico, cirurgia, curativo (local e tipo de cobertura), respectivamente, obtiveram-se os valores: $p=0,527 ; p=0,653 ; p=0,258$; $\mathrm{p}=0,610 ; \mathrm{p}=0,400 ; \mathrm{p}=0,416 ; \mathrm{p}=0,399 ; \mathrm{p}=278$; $p=151$. Assim, não houve associações estatisticamente significantes, com $p>0,05$.

\section{DISCUSSÃO}

As malformações congênitas apresentam em sua maioria a predominância do sexo masculino como os mais acometidos, com frequência maior que $50 \%$ de suas amostras, o que diverge deste estudo, uma vez que sobressaíram os recém-nascidos do sexo feminino $(63,3 \%)^{(2,10)}$.

No que se referiu ao peso de nascimento, o intervalo compreendido entre 2500 e 3999, considerado peso adequado, sobressaiu em $43,3 \%$ dentre os demais, porém ao somar os recém-nascidos que apresentaram peso inferior à $2500 \mathrm{~g}$, totalizaram-se $46,7 \%$ da amostra, com média ao nascimento de $2.726 \mathrm{~g} \pm 985.45$.

Em estudo que utilizou a classificação de peso $<2500 \mathrm{~g}$ e $\geq 2500 \mathrm{~g}$, evidenciou-se o nascimento de 10 e 56 neonatos malformados respectivamente, o que demonstra maior frequência do peso normal dentre recémnascidos $^{(11)}$.

Entretanto, há divergências quando se citam que fatores genéticos ou ambientais podem ser responsáveis pela presença de malformações congênitas e também estariam implicadas na gênese do baixo peso, explicando, desta forma, a associação entre a presença de malformações congênitas e o baixo peso ao nascer ${ }^{(12)}$.

Quanto à idade gestacional, a maior incidência de malformações ocorreu entre a $37^{a}$ e a $42^{a}$ semana de gestação, em percentual de $50 \%$ das gestantes pesquisadas. Na Turquia, pesquisa abordando apenas malformações do sistema gastrointestinal e doenças cardíacas congênitas, em que objetivou investigar a relação entre defeitos cardíacos congênitos e incidência e mortalidade em crianças com malformação do sistema gastrointestinal, com amostragem de 242 crianças, corroborou-se com este estudo, quando referiu à média de idades gestacionais das crianças de $37,2 \pm 2,6$ e peso ao nascer de $2756 \pm 612 \mathrm{~g}^{(13)}$. Em estudo realizado em Campina Grande, no nordeste do Brasil, com registro de 190 malformações congênitas no período de janeiro/2003 a dezembro/2005, mostrou-se que a maioria das mães apresentou idade gestacional entre 37 e 41 semanas e não demonstrou associação estatística entre idade gestacional e presença de malformação congênita $x^{2}=0,57$, $p=0,30^{(14)}$.

É importante discorrer sobre valores estatísticos das evidências científicas não apenas em nível de Brasil, como em todo o mundo, para que se possam enveredar conclusões pertinentes ao que se refere ao perfil de RN malformados.

Estudo realizado no Líbano, objetivando avaliar a incidência, os tipos e correlatos de anomalias congênitas em hospitais libaneses, comprovou-se o perfil dos recémnascidos, destacando o sexo masculino como o mais prevalente, com 58,3\% da amostra e $41,7 \%$ do sexo feminino; peso ao nascimento com média de

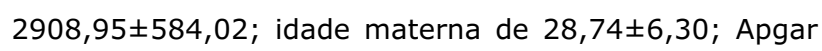
ao $5^{\circ}$ minuto de $5,71 \pm 3,63$, tendo o peso e o Apgar apresentado significância estatística com as malformações congênitas com $p=0,027$ e $p<0,001$, respectivamente ${ }^{(15)}$.

No que concerne à idade materna, neste estudo, sobressaíram os neonatos com mães entre 20 e 30 anos (60\%). No entanto, não foi encontrada significância estatística no cruzamento com as malformações. Outro estudo apresenta que $65(26,4 \%)$ das mães eram adolescentes, $148(60,2 \%)$ adultas e $33(13,4 \%)$ tardias. As chances de adolescentes, que tiveram gestações múltiplas, gerarem crianças com MC é de 6,14 vezes maiores que em adolescentes com gestação única. Todavia, a incidência em mães tardias é de 11,4 vezes maior, quando comparadas às mães com idade entre $20 \mathrm{e}$ 34 anos $^{(16)}$.

As malformações mais prevalentes foram as do sistema osteomuscular (17 RN) e do SNC (11 RN). A literatura apresenta resultados semelhantes quanto à prevalência de malformações do SNC e osteomuscular, em evidência de $31 \%{ }^{(2)}$. Outro estudo destacou altos índices de malformações dos sistemas nervoso e osteomuscular de 47,3 e $13,2 \%$ respectivamente, seguidos de fenda labial e/ou palatina, com $3,5 \%{ }^{(17)}$. 
Apesar de essas malformações apresentarem percentual elevado, aquelas com menor comprometimento e complexidade têm melhor prognóstico, enquanto as mais complexas necessitam de acompanhamento ambulatorial e intervenções para reestabelecimento funcional ou redução de sequelas. 0 diagnóstico das anomalias do sistema osteomuscular pode ser realizado durante a vida intrauterina, auxiliado por imagens ultrassonográficas. No entanto, faz-se necessária minuciosa avaliação por meio desse exame para que anomalias menores, cuja avaliação clínica demonstra normalidade, não passem despercebidas aos olhos dos avaliadores $^{(18)}$.

As anormalidades do SNC, segunda malformação com maior frequência dentre os diagnósticos deste estudo, refletem defeitos em todo o organismo, dependendo do local em que ocorram, sendo responsável pela formação de órgãos e funcionalidades orgânicas.

Neste estudo, a classificação que envolveu o SNC atingiu 22,4\%. Dados semelhantes foram evidenciados em pesquisa realizada em Recife, com 3.908 nascidos vivos, em que houve maior frequência nas anomalias do SNC $(27,4 \%)$ e osteomuscular $(21,2 \%)^{(16)}$.

Não foi possível analisar antecedentes familiares de malformações congênitas, o que inviabilizou a correlação entre a prevalência de malformações e os fatores de risco. Ressalta-se, também, que não foram analisados os casos de óbitos fetais ou natimortos por malformação, pois não tratavam de objetos da pesquisa.

No tocante às terapêuticas utilizadas com os RN malformados, pôde-se observar que estas diversificaram conforme o tipo de malformação, as necessidades apresentadas pelos neonatos e os riscos a que o RN estava exposto. Os cuidados dispensados ao RN com determinada anomalia individualmente, não são, necessariamente, os mesmos para todos que nascem com defeitos congênitos. Para efetivação do trabalho humanizado em Unidade Neonatal, é necessária equipe integrada, na qual a articulação das ações e saberes entre os diversos profissionais seja capaz de produzir a assistência integral aos neonatos, melhorando a qualidade da assistência prestada ${ }^{(19)}$.

A oxigenoterapia, enquanto terapêutica, é utilizada em todos os recém-nascidos que necessitam de suporte ventilatório para adaptação extrauterina ${ }^{(20)}$. Observou-se, neste estudo, que a terapia com oxigênio também foi administrada em RN com malformações que apresentavam relação direta com o sistema respiratório ou digestivo, devido ao comprometimento na ventilação espontânea do bebê. Nos casos de fenda palatina, as terapias instituídas voltaram-se à alimentação e ao suporte ventilatório, conforme necessidades.

Para sobrevivência de recém-nascidos de muito baixo peso e criticamente doentes, como os malformados, é imprescindível suporte nutricional adequado para suprir as demandas de crescimento e desenvolvimento desses neonatos $^{(21)}$. Para este estudo, os cuidados foram direcionados à prevenção de complicações que esse RN poderia desenvolver e ao seu quadro clínico.

A conduta mais comum frente à fenda palatina é a correção cirúrgica, executada em uma ou mais etapas e acompanhamento ambulatorial. Em casos de malformações, como gastrosquise e mielomeningocele, é sugerido tratamento cirúrgico nas primeiras 24 horas de vida, na tentativa de prevenir infecções ou complicações advindas da malformação, conduzindo a um menor tempo de oxigenoterapia e NPT, como redução do período de internação hospitalar ${ }^{(22-23)}$.

Ao referir sobre a realização de curativos, ressalta-se que constitui em procedimento necessário apenas a determinadas malformações que apresentam lesões significativas e aparentes, como no caso de gastrosquise, onfalocele, espinha bífida, principalmente lesões por mielomeningocele. Consoante aos resultados apresentados, o local dos curativos mais acometidos foi a região sacra, cuja associação esteve diretamente relacionada às malformações da espinha bífida.

Em geral, a mielomeningocele, malformação do SNC mais evidenciada, conforme dados descritos nos resultados, localiza-se na região lombossacra da coluna vertebral. O líquido cefalorraquidiano pode escapar através do defeito, em que, muitas vezes, é coberto por um fino saco membranoso, caso contrário, o tecido fica exposto(22).

Para realização de cuidados de enfermagem à lesão por mielomeningocele, é necessário controlar a pressão sobre áreas do corpo, observar as características da lesão, aplicar solução adequada à pele/lesão, quando apropriado e realizar curativo adequado ${ }^{(24)}$. O curativo dessas lesões varia segundo a rotina das Unidades Neonatais nas instituições hospitalares e a disponibilidade das coberturas e dos materiais adequados, consoante às características da própria lesão.

Portanto, por meio dos diagnósticos das malformações, das terapias sofisticadas, dos procedimentos cirúrgicos, dos aparelhos e dispositivos modernos e profissionais especializados, têm-se garantida chance de vida a recém-nascidos que há algumas décadas eram considerados inviáveis pela ciência ${ }^{(25)}$. 


\section{CONCLUSÃO}

Algumas malformações requerem cuidados $e$ terapêuticas específicas, conforme evolução clínica do RN diante da anomalia apresentada, no entanto não estão diretamente relacionadas ao tipo de malformação. Contudo, o quadro clínico que o neonato apresenta poderá assemelhar-se ao de qualquer outro RN sem defeitos congênitos.

Dentre as terapêuticas mais utilizadas, ressaltam-se a oxigenoterapia, antibioticoterapia, a terapia nutricional por meio da sonda orogástrica e os curativos com colagenase, no entanto não apresentaram associações estatisticamente significantes com as malformações congênitas.

Portanto, outros estudos com amostra em maior número são necessários para averiguar possível associação entre as terapêuticas utilizadas em RN e as malformações congênitas. Embasados em evidências científicas, buscou-se contribuir para que os profissionais da enfermagem, bem como a comunidade acadêmica, possam adquirir conhecimentos referentes à prática hospitalar instituída aos recém-nascidos com necessidades especiais, visando aprimorar a assistência a ser desenvolvida de maneira sistematizada e individualizada, proporcionando melhoria da qualidade de vida e promovendo a saúde desses recém-nascidos em Unidade Neonatal, visto que a maioria dos estudos publicados está voltada à prevalência de malformações e não especificamente à assistência de Enfermagem implementada a essa população específica.

10. Pereira RJS, Abreu LC, Valenti VE, Albuquerque WDM, Pereira $\mathrm{SC}$, Araújo R, et al. Freqüência de malformações congênitas das extremidades em recém-nascidos. Rev. bras. crescimento desenvolv. hum. [Internet]. 2008 [acesso em: $31 \mathrm{mar}$ 2015];18(2):155-62. Disponível em:

http://pepsic.bvsalud.org/scielo.php?script=sci_arttext\&pid=S01 04-12822008000200006.

11. Melo WA, Zurita RCM, Uchimura TT, Marcon SS. Anomalias congênitas: fatores associados à idade materna em município sul brasileiro, 2000 a 2007. Rev. Eletr. Enf. [Internet]. 2010 [acesso em: 31 mar 2015];12(1):73-82. Disponível em: http://dx.doi.org/10.5216/ree.v12i1.5994.

12. Cunha CJ, Fontana T, Garcias GL, Martino-Roth MG. Fatores genéticos e ambientais associados a espinha bífida. Rev. Bras. Ginecol. Obstet. [Internet] 2005 [acesso em: 31 mar 2015];27(5):268-74. Disponível em: http://dx.doi.org/10.1590/S0100-72032005000500007. 13. Orun UA, Bilici M, Demirceken FG, Tosun M, Öcal B, Cavusoglu $\mathrm{YH}$, et al. Gastrointestinal system malformations in children are associated with congenital heart defects. Anadolu Kardiyol Dergisi [Internet]. 2011 [acesso em: $31 \mathrm{mar}$ 2015];11(2):146-9. Disponível em:

http://dx.doi.org/10.5152/akd.2011.034.

14. Brito VRS, Sousa FSD, Gadelha FH, Souto RQ, Rego ARDF, França ISXD. Malformações congênitas e fatores de risco materno em Campina Grande-Paraíba. Rev Rene [Internet]. 2010 [acesso em: 31 mar 2015];11(2):27-36. Disponível em: http://www.revistarene.ufc.br/revista/index.php/revista/article/vi ew/370.

15. Francine R, Salameh PS, Aline H. Congenital Anomalies: Prevalence and Risk Factors. Univ J Public Health. 2014; 2(2):5863.

16. Amorim MMR de, Vilela PC, Santos ARVD, Lima ALMV, Melo EFP de, Bernardes HF, et al. Impacto das malformações congênitas na mortalidade perinatal e neonatal em uma maternidade-escola do Recife. Rev Bras Saúde Matern Infant [Internet]. 2006 [acesso em: 31 mar 2015];6(Suppl 1):s19-25. Disponível em: http://dx.doi.org/10.1590/S151938292006000500003.

17. Pinto CO, Nascimento LFC. Estudo de prevalência de defeitos congênitos no Vale do Paraíba Paulista Rev Paul Pediatr [Internet] 2007 [acesso em: 31 mar 2015]; 25(3):233-9. Disponível em: http://dx.doi.org/10.1590/S010305822007000300007.

18. Noronha Neto C, Souza ASR, Moraes Filho OB, Noronha AMB. Validação do diagnóstico ultrassonográfico de anomalias fetais em centro de referência. Rev Assoc Med Bras [Internet]. 2009 
[acesso em: 31 mar 2015];55(5):541-6. Disponível em: http://dx.doi.org/10.1590/S0104-42302009000500016. 19. Silva LG, Araújo RT, Teixeira MA. O cuidado de enfermagem ao neonato pré-termo em unidade neonatal: perspectiva de profissionais de enfermagem. Rev. Eletr. Enf. [Internet]. 2012 [acesso em: 31 mar 2015];14(3):634-43. Disponível em: http://dx.doi.org/10.5216/ree.v14i3.12531.

20. Brasil TB, Barbosa AL, Cardoso MVLML. Aspiração orotraqueal em bebês: implicações nos parâmetros fisiológicos e intervenções de enfermagem. Rev Bras Enferm [Internet]. 2010 [acesso em: 31 mar 2015];63(6):971-7. Disponível em: http://dx.doi.org/10.1590/S0034-71672010000600016. 21. Costa P, Kimura AF, Vizzotto M de PS, Castro TE de, West A, Dorea E. Prevalência e motivos de remoção não eletiva do cateter central de inserção periférica em neonatos. Rev Gaúcha Enferm [Internet]. 2012 [acesso em: 31 mar 2015];33(3):12633. Disponível em: http://dx.doi.org/10.1590/S198314472012000300017.

22. Veir Z, Duduković M, Miklić $P$, Mijatović $D$, Cvjeticanin B, Veir $M$, et al. Reconstruction of a Soft Tissue Defect of the Back. Coll Antropol. 2011;35(4):1295-8.

23. Alali JS, Tander B, Malleis J, Klein MD. Factors affecting the outcome in patients with gastroschisis: how important is immediate repair? Eur J Pediatr Surg [Internet]. 2011 [acesso em: 31 mar 2015];21(2):99-102. Disponível em: http://dx.doi.org/10.1055/s-0030-1267977.

24. Gurgel E de PP, Rolim KMC, Galvão MTG, Caetano JÁ. Abordagem assistencial ao neonato portador de mielomeningocele segundo o modelo de adaptação de Roy. Rev Esc Enferm USP [Internet]. 2010 [acesso em: $31 \mathrm{mar}$ 2015];44(3):702-7. Disponível em: http://dx.doi.org/10.1590/S0080-62342010000300021. 25. Silva LJ, Silva LR, Christoffel MM. Tecnologia e humanização na Unidade de Terapia Intensiva Neonatal: reflexões no contexto do processo saúde-doença. Rev Esc Enferm USP [Internet]. 2009 [acesso em: 31 dez 2015];43(3):684-9. Disponível em: http://dx.doi.org/10.1590/S0080-62342009000300026.

Artigo recebido em 08/03/2013.

Aprovado para publicação em 05/06/2014.

Artigo publicado em 31/03/2015. 